Développement des territoires et communication : politiques et pratiques à l'œuvre

\title{
Associations et construction européenne : vers une société civile internationale?
}

Associations and European Construction: Toward an International Civil Society?

\section{Eric Dacheux}

\section{(2) OpenEdition}

\section{Journals}

Édition électronique

URL : http://journals.openedition.org/edc/120

DOI : $10.4000 /$ edc. 120

ISSN : 2101-0366

Éditeur

Université Lille-3

Édition imprimée

Date de publication : 1 décembre 2003

ISSN : 1270-6841

Référence électronique

Eric Dacheux, « Associations et construction européenne : vers une société civile internationale ?»,

Études de communication [En ligne], 26 | 2003, mis en ligne le 13 octobre 2008, consulté le 22 avril

2019. URL : http://journals.openedition.org/edc/120 ; DOI : 10.4000/edc.120

Ce document a été généré automatiquement le 22 avril 2019.

(c) Tous droits réservés 


\title{
Associations et construction européenne : vers une société civile internationale?
}

Associations and European Construction: Toward an International Civil Society?

\author{
Eric Dacheux
}

En ce début de XXIe siècle, les manifestations de Porto Alegre, Gêne, Nice ou Barcelone remettent la notion de société civile au goût du jour. Dans le même temps, les associations sont l'objet de toutes les sollicitudes de la part des institutions politiques. En France, dans le cadre des célébrations du centenaire de la vie associative, le gouvernement français a créé un site Internet consacré à la vie associative ${ }^{1}$ et a co-signé avec la Conférence permanente des coordinations associatives ${ }^{2}$ une charte de la vie associative, prévoyant les engagements réciproques du monde associatif et de l'administration publique. Les institutions européennes ne sont pas en reste, puisque les associations sont au centre du livre blanc sur la « nouvelle gouvernance européenne » paru en juillet 2001. De plus, pour palier au déficit démocratique de l'Europe, se met lentement en place un «dialogue civil » européen entre les institutions européennes et les réseaux associatifs européens. Nous reviendrons sur ce point dans la quatrième et dernière partie de ce texte. Dans un premier temps, nous dresserons un rapide panorama de la vie associative en Europe, en insistant sur le lien fondamental qui unit la construction d'une communauté politique nationale et le développement de la vie associative. Nous définirons, dans un deuxième temps, le cadre théorique de notre analyse. Dans un troisième temps, nous présenterons notre objet étude, les réseaux associatifs européens. Enfin, dans une dernière partie, nous analyserons le rôle que peuvent jouer ces réseaux associatifs dans la construction d'une Union politique européenne. Ce qui nous permettra, en conclusion, de discuter la thèse en vogue de l'émergence d'une société civile internationale. Cependant, avant d'aborder la première partie de cette étude, il convient d'apporter quelques précisions méthodologiques. Quatre outils ont été mobilisés dans cette recherche débutée en septembre 1998 : 
L'Analyse de contenu de divers documents associatifs (plaquettes, newsletters, etc.) mais aussi de sites Internet.

Une vingtaine d'entretiens semi-directifs conduits auprès de responsables associatifs et de fonctionnaires européens.

L'observation participante à douze rencontres européennes organisées par des réseaux associatifs européens dans trois pays différents (France, Belgique, PaysBas).

La conduite de trois séminaires pluridisciplinaires mêlant chercheurs, responsables associatifs, fonctionnaires européens et élus de nationalités différentes. Ces trois séminaires permettant, d'une part, d'avoir une vision élargie des rapports entre associations et construction européenne et, d'autre part, d'observer in vivo le fonctionnement d'un espace public européen restreint.

\section{Diversité de la vie associative dans l'union européenne}

2 La question associative est trop souvent saisie dans un cadre national. L'Europe offre la possibilité de sortir de ce cadre et de développer des analyses comparatives. En théorie du moins, car de telles analyses, au niveau de l'Union européenne, sont lourdes, complexes et ne peuvent pas s'appuyer sur des données fiables : les chiffres dont on dispose ne sont souvent que des estimations, des approximations qui peuvent varier du simple au triple. Cependant, des travaux internationaux permettent de se faire une première image de la vie associative dans les pays membres de l'Union européenne. Or, on est frappé lorsqu'on lit, l'enquête diligentée par la Commission européenne (1997), celle effectuée par le Haut conseil de la coopération internationale (Doucin, 2000) ou celle conduite sous l'égide de l'université Johns Hopkins (Salamon, Anheier, 1994), par la disparité de la vie associative européenne. Cette grande hétérogénéité se marque par la définition même de l'association: sept pays sur les quinze que comptent l'Union ne possèdent pas de définition juridique de l'association ${ }^{3}$, tandis qu'il n'existe toujours pas, au niveau européen, une définition juridique de l'association européenne. Mais cette diversité se retrouve également dans le nombre d'associations par pays : 0,5 associations pour 1000 habitants au Luxembourg, 4,3/1000 en Allemagne, 9/1000 en Autriche, 12,4/1000 en France, 22,4/1000 en Finlande. Hétérogénéité, encore, dans le régime fiscal applicable aux associations et dans le nombre de personnes nécessaires pour constituer une association ${ }^{4}$. Pluralité, toujours, dans les problèmes mis en avant par les dirigeants associatifs européens. À la question : quels sont pour vous les principaux défis du XXIe siècle ? Les responsables espagnols répondaient : l'adaptation à la modernisation sociale; les italiens : l'autofinancement; les français: la montée de l'individualisme; les allemands la diversification des ressources financières 5 ! Disparité, enfin, dans le poids respectif des différents secteurs associatifs : les associations d'actions sociales dominent en Belgique (15\%), mais en France ce sont les associations sportives qui dominent $(127000){ }^{6}$, alors qu'en Finlande le premier secteur associatif est constitué par les associations professionnelles, tandis qu'en Grande-Bretagne le secteur caritatif est - de loin! - le plus important avec plus de 116000 Charities (sur 400000 associations)!

3 Cette liste des différences profondes existant entre les mondes associatifs nationaux des différents pays des quinze est loin d'être exhaustive. Néanmoins, elle montre que la diversité du droit des associations dans les pays de l'Union européenne n'est pas une aberration technocratique, mais le reflet de la diversité des situations nationales. La vie associative est profondément dissemblable d'un pays à l'autre, parce que la vie sociale et politique diffère profondément d'un pays européen à l'autre. Autrement dit, il faut 
considérer le lien entre construction d'une communauté politique nationale et configuration du monde associatif comme un lien fort, un lien charnel. La variété de la vie associative en Europe est le reflet de la diversité des chemins ayant conduit les pays de l'Union vers la démocratie. On peut justifier ce lien entre démocratie et vie associative de manière empirique en remarquant, par exemple, que dans l'histoire récente des pays comme la France, l'Espagne, l'Allemagne ou l'Italie, la venue au pouvoir des régimes autoritaires et fascistes s'est traduite par une restriction considérable de liberté associative ou que, à l'inverse, depuis la chute du Mur, les pays d'Europe centrale et orientale connaissent un fort développement de la vie associative. On peut également essayer de justifier ce lien de manière plus théorique, c'est l'objet de la seconde partie.

\section{L'association : un acteur de la société civile participant à la construction de l'espace public}

4 La réalité associative est mouvante et contrastée. De plus, elle est saisie par des disciplines différentes (sciences politiques, sociologie, histoire, etc.) qui ne mettent pas forcément les mêmes définitions sous les mêmes mots. C'est pourquoi, nous allons maintenant donner la définition de trois termes clefs : société civile, espace public et association, non pas dans une perspective normative, mais dans le but de faciliter le débat critique autour de notre travail.

\section{Société civile}

5 Cette notion s'avère d'un maniement délicat. D'une part, elle s'apparente, comme le souligne François Rongeon (1986), à un mythe politique dans la mesure où elle est invoquée par différents acteurs (élus, fonctionnaires, syndicalistes, dirigeants économiques, responsables associatifs, etc.) qui ne mettent pas forcément les mêmes valeurs sur ce terme socialement valorisé. Certains, dans une perspective libérale, valorisent l'autonomie, la responsabilité individuelle, l'émancipation de l'État, tandis que d'autres y voient un principe d'action collective, une solidarité réciprocitaire capable de contrebalancer les mécanismes d'exclusion du marché. En dehors de cette ambiguïté idéologique, l'utilisation du mot «société civile» est, d'autre part, rendue malaisée en raison des différentes acceptions que ce terme a connues au cours de l'histoire. En effet, la société civile renvoie, chez Aristote, à la société politiquement organisée opposée à la société sauvage. Dans ce sens, État et société civile sont quasiment synonymes. Mais ce terme, depuis la révolution française, désigne ce qui relève du domaine privé et qui s'oppose à l'État. Dans cette acception, le mot société civile peut donc désigner tous les rapports sociaux et économiques hors État. Dans une acception plus récente et plus restreinte qui est devenue la norme, le mot société civile recouvre les interactions sociales hors sphère économique et sphère étatique. C'est cette dernière acception que nous ferons notre en précisant cependant trois éléments :

Dans la réalité empirique, les individus conjuguent avec plus ou moins de bonheur leurs différentes identités sociales : une même personne peut être, tout à la fois, fonctionnaire, militant associatif et consommateur. Il n'y a donc pas de séparation radicale entre société civile, système étatique et marché, mais interaction entre ces trois sphères constitutives du monde commun (Laville, 2001).

La société civile est celle des engagements collectifs des individus. Or, ces engagements se font dans de nombreuses organisations: groupes professionnels, 
coordinations, mutualités, ONG, etc. Autrement dit, il n'y a pas équivalence entre association et société civile.

Comme le rappelle, à la suite de Walzer, Philippe Chanial, il existe un lien étroit entre vitalité de la société civile et vitalité de la démocratie. L'inverse est également vrai, sans un état démocratique favorisant l'engagement libre et volontaire des individus dans des organisations de leurs choix, il n'y a pas de société civile dynamique. Comme le dit l'auteur: "Parce que la société civile est marquée tout à la fois par l'inégalité, la fragmentation et la discontinuité, elle ne pourra accomplir sa mission - sauver l'État démocratique - sans l'aide de l'État qu'elle est censée sauver » (Chanial, 2001, p. 157).

\section{Espace public}

Conceptualisée par Kant et popularisée par Habermas, la notion d'espace public est parfois décriée en raison de son fort contenu normatif et de son faible ancrage historique et empirique (Schudson, 1992). Néanmoins, elle est utilisée dans de nombreuses disciplines des sciences humaines, en prenant, bien souvent, des acceptions différentes voire contradictoires. Pour notre part, nous nous centrons sur l'acception politique de cette notion. Selon nous l'espace public est tout à la fois :

Un espace de médiation entre société civile, système étatique et marché.

Le lieu de légitimation du politique. C'est par l'espace public que les citoyens ont accès aux informations politiques, qu'ils peuvent débattre et se forger une opinion et qu'ils peuvent choisir les personnes qui exerceront le pouvoir politique. C'est par l'espace public que les citoyens se sentent non seulement destinataires du droit, mais aussi auteur de ce droit.

Un espace symbolique qui, comme le rappelle le philosophe Etienne Tassin (1991), permet de relier entre eux des individus n'appartenant plus à des communautés traditionnelles. L'espace public est le fondement de la communauté politique.

7 Cette définition appelle quelques remarques et précisions :

L'espace public ne se réduit pas à l'espace politique institutionnel.

L'espace public est un concept unificateur qui dans la réalité empirique est composé d'une multitude d'espaces publics plus ou moins larges, plus ou moins autonomes du système étatique ou du système marchand.

L'espace public est un espace de communication. Cette communication est parfois orientée vers l'intercompréhension comme le souligne Habermas (1987), mais aussi comme le rappelle les critiques du philosophe allemand (Calhoun, 1992 ; François, Neveu, 1999), elle vise à la persuasion et emprunte alors souvent les chemins de la séduction.

L'espace public est un espace d'action. L'espace public est un espace où les citoyens organisés collectivement tentent de mobiliser l'opinion, font part de leurs protestations et émettent des propositions.

\section{Association}

8 L'association doit se comprendre comme une organisation en tension. Tout d'abord, comme le signalait Tocqueville, tension entre l'individu et le collectif, la liberté et l'égalité, ce qui fait de toute association un rouage essentiel de la démocratie. Pour le dire autrement, les associations modernes sont toutes des acteurs politiques car elles permettent de concilier différence et appartenance. Conciliation qui est au cœur même du projet démocratique. Toutefois, il est évident que le club d'échec de St Étienne ne joue pas, dans nos démocraties, le même rôle que MSF. C'est pourquoi, nous centrons nos propos sur des organisations non lucratives qui sont créées par des citoyens et qui 
poursuivent des buts d'intérêts généraux. Ce type bien particulier d'associations que Bruno Rebelle (Rebelle, Swiatly, 1999) nomme les «Forces agissantes» du monde associatif n'est pas majoritaire d'un poids de vue quantitatif. Pourtant, implicitement, il constitue le noyau de référence de nombreuses recherches. L'observation montre que ce type d'association se situe entre deux pôles. D'une part, les associations peu professionnalisées, peu ou pas ancrées dans le marché et qui se placent délibérément dans le champ politique. Ce type d'association à l'image d'Act up, Attac, ou Résistance à l'agression publicitaire auront tendance à se penser comme étant des contre-pouvoirs, des forces d'opposition. D'autre part, des associations fortement professionnalisées, présentes sur le marché et assurant des prestations visant à réparer le système social. Ces associations, la plupart du temps gestionnaires de structures culturelles ou hospitalières, auront tendance à se penser comme des partenaires, des forces de proposition.

Pour résumer les propos de cette seconde partie, nous dirons que la société civile ne se réduit pas au monde associatif qui ne se réduit pas aux organisations non lucratives d'intérêt général. De même, l'espace public, élément fondateur de la démocratie, est un espace de communication et d'action politique qui ne se limite pas aux interactions entre journalistes, hommes politiques et sondeurs. Enfin, les associations que nous étudions sont issues de la société civile et jouent un rôle démocratique essentiel : en conciliant différence et appartenance, elles participent à la constitution d'une communauté politique. Comme nous l'avons vu dans la première partie, le développement de ces associations dans les différents pays de l'Union européenne n'est pas homogène, il n'obéit pas à un mécanisme simple et unique comme l'incomplétude de l'État social ou la prégnance de l'Église. Il est intimement lié à la marche du pays vers la démocratie, c'est d'ailleurs pourquoi Tocqueville fait de la science des associations la science mère de la démocratie. Dès lors, dans ce cadre d'analyse qui fait de l'association issue de la société civile un des acteurs centraux de la constitution de l'espace public de l'État-nation, il est tentant d'élargir le point de vue : si le lien entre développement de la vie associative et constitution d'une communauté nationale démocratique est consubstantiel, qu'en est-il du lien entre européanisation de la vie associative et création d'une communauté politique de taille européenne? Autrement dit, l'émergence de réseaux associatifs européens peut-elle contribuer à l'émergence d'un espace public européen? C'est l'objet de notre quatrième et dernière partie, mais auparavant, il nous faut définir ce que nous entendons par « Réseaux associatifs européens ».

\section{Les réseaux associatifs européens}

10 Dès le début du XXe siècle, des associations militant pour la construction d'une Europe politique se sont constituées en réseaux européens. La plus célèbre étant sans doute l'Union pan européenne du Comte Richard de Coudenhove-Kalergi (Réau du, 1995). Un siècle plus tard, les réseaux associatifs européens sont nombreux et déploient d'intenses efforts de lobbying à Bruxelles. Bien entendu, nos recherches ne portent pas sur tous ces réseaux associatifs. Tout d'abord, nous avons étudié les réseaux associatifs internes à l'Union européenne et non les réseaux associatifs extérieurs qui, comme le souligne le livre blanc sur la gouvernance de l'Union européenne (Commission européenne, 2001), jouent un rôle non négligeable dans la politique extérieure de l'Union européenne. De plus, nous centrerons nos propos sur les réseaux civiques, écartant de l'analyse les associations professionnelles européennes qui, pourtant, sont des experts très influents 
lors de la mise en place de normes européennes spécialisées. Enfin, nous n'avons pas étudié les relations entre les réseaux civiques et l'ensemble des institutions européennes, mais entre ces réseaux et trois instances : le Parlement européen, la Commission et le Comité Economique et Social européen. Loin de décrire les rapports entre ce que les institutions européennes appellent "la société civile organisée au niveau européen » (Comité économique et social européen, 1999) et l'Union européenne, nous menons des recherches sur le répertoire d'action et de communication d'une minorité de réseaux associatifs européens en direction de trois instances spécialisées de nature fédérale.

11 On peut définir deux grands types d'associations européennes civiques : celles, généralement les plus anciennes, qui se présentent sous la forme d'une organisation fortement institutionnalisée, aux contours stables, le plus souvent une fédération européenne, composées de fédérations nationales. C'est le cas du Mouvement européen, des Fédéralistes européens, de la Fédération européenne des jeunes écologistes, etc. Mais, de plus en plus, comme le note Julien Weisbein (2000), l'européanisation des associations se développe sous une forme réticulaire. Par réseaux associatifs européens, il faut comprendre un ensemble d'associations disparates, allant de l'association locale à l'ONG mondiale, liées entre elles par un objectif commun, mais dont les normes et les contours sont peu définis. On peut citer, par exemple, les Conférences inter citoyennes (CIC), le Forum civique européen ou Euro Citizen Action Service. Cependant, ces différences organisationnelles n'empêchent pas ces deux types d'associations européennes de travailler ensemble sur des opérations ponctuelles ou de se regrouper dans une structure permanente. Si bien que l'on peut étendre l'appellation «réseaux associatifs » à l'ensemble des associations européennes civiques. Ces réseaux associatifs européens civiques ne sont pas déconnectés des autres mouvements sociaux européens. Au contraire, sous l'impulsion du Mouvement européen international, 17000 organisations (locales, nationales, européennes ou mondiales) se sont regroupées au sein du « Forum Permanent de la Société civile ». Forum où se côtoient des organismes aussi différents que la Confédération européenne des syndicats, le Bureau européen de l'environnement ou Amnesty International. À l'intérieur de ce forum, les associations européennes civiques ${ }^{7}$ sont moins de 500 et regroupent environ 40000 personnes, en tout cas si l'on s'en tient aux chiffres - bien incomplets - fournis par le Forum. Une autre estimation (Balme, Chabanet, 2002), faite à partir d'une base de données officielle de l'Union européenne recensant les différents lobbies européens, donne des chiffres encore plus modestes ${ }^{8}$. Malgré cette faiblesse numérique, nous allons voir maintenant que les réseaux associatifs civiques sont choyés par les instances européennes.

\section{Rôle des réseaux associatifs européens dans la construction politique de l'union}

12 Plus l'Europe devient concrète, moins le processus politique d'unification rencontre de soutien. Ce paradoxe, mis en lumière par le taux d'abstention croissant aux élections européennes, est lié - tous les acteurs le soulignent - à la faible légitimité des instances européennes. Ce problème de légitimité est, en effet, relevé par les chercheurs (Boual, 1999 ; Wolton, 1993), les responsables associatifs européens (Promothéus Europe, 1999 ; Peugeot, 1999) et les instances européennes elles-mêmes (Parlement européen, 1996; Commission européenne, 1997b). Or, nous l'avons vu, l'espace public est, en démocratie, le lieu de légitimation du politique. En l'absence d'un espace public européen large et 
populaire, la construction politique de l'Europe souffre donc d'un important déficit de légitimité : "Ce qui manque le plus, c'est un dialogue européen ascendant et interactif " souligne ainsi le rapport du comité économique et social européen (2000). «Il s'agit de créer un 'espace' transnational dans lequel les citoyens de divers pays peuvent débattre de ce qu'ils considèrent comme les défis majeurs de l'Union. Les décideurs politiques devraient ainsi pouvoir rester en contact avec l'opinion publique européenne, et ce dialogue pourrait leur indiquer quels projets européens suscitent l'adhésion de la population » renchérit le livre blanc sur la gouvernance (Commission européenne, 2001). C'est pourquoi, ce même livre blanc cherche les moyens concrets de mettre en place un dialogue supranational pouvant accélérer l'émergence d'un espace public européen. Il suggère deux pistes principales : d'une part, le développement des nouvelles technologies de la communication (forum de discussion sur Internet, mise en ligne des documents soumis à la discussion, etc.) ; d'autre part, la mise en place d'un dialogue institutionnalisé entre les instances européennes et les ONG (Commission européenne, 2001). À côté du dialogue social qui concerne les organisations syndicales et patronales européennes, la Commission entend, en effet, développer un " dialogue civil européen ", c'est-à-dire, une concertation ouverte aux associations et fondations de manière à ce que les décisions prises ne soient plus perçues comme étant le produit d'un obscur cheminement technocratique unilatéral, mais comme le fruit d'un processus transparent de débats entre les institutions et les représentants de la société civile européenne. Dans ce contexte, la faiblesse numérique des réseaux associatifs civiques européens, importe moins que leur capacité de légitimation du système politique européen. Effectivement, les réseaux associatifs européens présentent un soutien précieux au processus de construction de l'Europe politique. Et ce, pour cinq raisons au moins :

Elles constituent des relais entre les citoyens et les institutions de l'Union. C'est ainsi que le service d'orientation téléphonique «l'Europe en direct» est cogéré, depuis 1996, par une demi-douzaine de réseaux associatifs européens qui, grâce aux fonds alloués par la Commission, s'efforcent, dans leur domaine respectif ${ }^{9}$, de répondre, dans un langage non technocratique, aux interrogations des européens (Weisbein, 2000).

Elles participent activement à la définition de certaines politiques publiques européennes. Par exemple, le réseau européen de lutte contre la pauvreté (REALPES), qui rassemble des associations comme Caritas Europe ou ATD Quart monde, est

régulièrement invité aux réunions de la Commission des affaires sociales du Parlement européen. Certaines Directions générales comme la DG «environnement» ont développé des relations suivies avec les ONG de leurs secteurs.

Elles sont impliquées dans des espaces de concertation entre la société civile et les institutions. Actuellement, dans le cadre du «Dialogue civil», les institutions européennes organisent des forums et conventions qui sont des espaces d'expression où des élus et fonctionnaires européens confrontent leurs points de vue avec des chercheurs et des représentants des réseaux associatifs européens. En témoignent les deux forums européens sur la politique sociale organisés (en 1996 et 1998) par la Commission européenne ou la récente «Convention sur le rôle et la contribution de la société civile organisée dans la construction européenne » mise en œuvre par le Comité économique et social (Bruxelles, octobre 1999).

Elles font émerger des espaces publics autonomes de taille européenne. Les associations européennes créent leurs propres espaces publics européens de manière à ce que des citoyens européens puissent développer, sans langue de bois, un débat européen sur des questions européennes. Par exemple, les Conférences intercitoyennes (CIC), réseaux regroupant une trentaine d'associations 
européennes à vocation civique, se réunissent tous les six mois, sur l'initiative de l'un des membres du réseau, afin de faire naître des discussions citoyennes pouvant faire contrepoids aux discussions technocratiques menées dans le cadre des conférences intergouvernementales $(\mathrm{CIG})^{10}$.

Ce sont des sources d'Utopie. Les réseaux européens multiplient les projets de constitutions, de chartes européennes. Elles portent en elles le projet d'une Europe sociale plus égalitaire et d'une Europe démocratique plus participative. Cette fonction utopique nous paraît fondamentale et c'est sur ce point que nous voudrions insister.

13 En effet, l'utopie est l'un des moteurs de l'action collective et, plus généralement, de l'action politique. Constat illustré par l'Union européenne elle-même, puisque, en définitive, l'histoire de la construction européenne est aussi celle de la lente concrétisation d'une utopie. Cependant, le rôle de l'utopie ne se limite pas à son pouvoir performatif. L'utopie est, dans nos démocraties, au fondement même du politique. En effet, nous pensons, avec Claude Lefort (1986), que la crise de la représentation que rencontre l'Europe est consubstantielle à la démocratie. En effet, comme le précise Lefort, la démocratie est: "un régime fondé sur la légitimité d'un débat sur le légitime et l'illégitimité ». La démocratie est un régime qui aménage une représentation du pouvoir en faisant un lieu vide qui n'appartient à personne. C'est pourquoi, «la démocratie se révèle ainsi la société historique par excellence, société qui, dans sa forme accueille et préserve l'indétermination ». Dès lors, l'étude des associations dans l'espace public s'inscrit dans un cadre théorique mettant l'accent, non pas sur une crise de la représentation de toute façon inhérente à la démocratie, mais sur la soumission du politique à l'économique, ce que Karl Polanyi (1983) nomme le désencastrement de l'économie. Pour le dire autrement, ce qui est frappant, ce n'est pas tant la remise en cause de la représentation que sa soumission à une idéologie instaurant le primat de l'économique sur le politique. Or, dans cette optique, la notion d'utopie est fondamentale, puisque, justement, l'utopie permet de lutter contre l'hégémonie de l'idéologie. C'est, en tout cas, ce que montre Paul Ricœur dans son livre «L'idéologie et l'Utopie » (Ricœur, 1997). Selon cet auteur, l'utopie est, "le remède à la pathologie de la pensée idéologique». En effet, poursuit l'auteur, "l'utopie nous met à distance de la réalité présente, elle nous donne l'aptitude à éviter de percevoir ladite réalité comme naturelle, nécessaire ou inéluctable, mais plus important encore l'utopie nous propose de nouvelles réalités possibles » (Ricœur, 1997).

Les réseaux associatifs européens de transformation sociale proposent, eux aussi, de «nouvelles réalités possibles ", puisqu'ils militent pour une Europe plus démocratique et tentent de faire advenir cet espace public européen qu'ils appellent de leurs vœux. Ainsi, selon nous, l'influence et l'importance effective des associations européennes ne tient pas dans leurs capacités - réelles mais somme toute assez limitées - à peser sur le processus institutionnel, ou à incarner une société civile dont elles ne sont guère représentatives. Mais cette influence politique tient à deux phénomènes empiriques et symboliques. Premièrement, au niveau empirique les associations européennes favorisent la naissance de communautés politiques élargies. La démocratie, rappelle Philippe Chanial, c'est le saut vers l'inconnu, l'invention nécessaire « d'un monde commun entre étrangers, d'une communauté de destin entre inconnus » (Chanial, 1998, 38). L'association permet de faire ce saut puisqu'elle donne naissance à un contexte commun permettant de nouer des liens de confiance Les associations européennes de transformation sociale favorisent, à leur échelle, un pari qui est aussi celui de l'Union européenne : la construction d'une communauté de destin avec des inconnus n'appartenant pas à la même nation. 
Deuxièmement, au niveau symbolique ; les associations européennes sont, des créatrices d'utopie. Par delà leur faiblesse numérique, leurs divisions idéologiques et leur dépendance à l'égard des financements européens, les réseaux civiques sont portés par un projet politique utopique : rendre le monde meilleur qu'il n'est. Cette visée prométhéenne est également celle de la démocratie. Comme le montre avec force Tocqueville, l'égalité est, avec la liberté, le moteur de la démocratie. En défendant et en diffusant des valeurs autres que celles de la lutte de chacun contre tous, les associations de transformation sociale révèlent que la raison instrumentale n'a pas vocation à régenter l'ordre social et politique. Pour ces deux raisons, nous pensons que les associations européennes constituent l'embryon d'un espace public aux dimensions de l'Europe. Pour autant, ce mouvement d'internationalisation des associations est-il le signe tangible de l'émergence d'une société civile internationale qui viendrait contrebalancer des pouvoirs économiques mondiaux ? La réponse à cette question servira de conclusion à ce texte.

Nos travaux montrent que l'européanisation du secteur associatif est en marche. Or, selon les analystes, cette européanisation d'un secteur de la société civile est vue, soit comme l'amorce de la constitution d'un espace public aux dimensions de l'Union (Weisbein, 2002; Mercier, 2003), soit comme le signe d'un mouvement plus profond qui touche tous les continents : la naissance d'une société civile mondiale (Caillé, 2001 ; Massiah, 2002). Nos observations empiriques et nos références théoriques nous conduisent à privilégier la première hypothèse. Elle nous semble corroborée par les faits, tandis que la seconde nous paraît relever d'une prospective hasardeuse. En effet, premier point, sur le terrain, les actions initiées par les réseaux associatifs européens pour construire un espace public de dimension européenne tendent à rejoindre celles des institutions européennes. Ce double mouvement (ascendant et descendant) de la société civile et de la sphère institutionnelle est accompagné par les acteurs économiques qui tirent généralement profit du marché unique. De plus, il s'ancre dans un espace juridique commun et s'accompagne de la lente émergence d'une socialité européenne (tourisme, travail transfrontalier, jumelages, échanges européens d'étudiants, etc.) et d'une symbolique européenne (drapeau, Euro, devise, etc.) pouvant donner naissance à un sentiment d'appartenance à une même communauté de destin. Bien sûr, tous ces phénomènes sont récents, minoritaires et réversibles mais, et c'est le deuxième point de notre raisonnement, cette hypothèse demeure, malgré tout, plus plausible que celle prédisant l'avènement d'une société civile mondiale. Effectivement, malgré l'importance croissante du mouvement altermondialisation, nous ne croyons pas à l'émergence d'une société civile mondiale, en tout cas au sens que nous avons donné à ce terme dans cet article ${ }^{11}$. Plusieurs faits nourrissent ce scepticisme. Tout d'abord, la fragilité et la réversibilité des processus en cours. Nous sommes, la guerre en Irak vient encore de le montrer, dans des phases de profondes turbulences où les opinions publiques (américaines, européennes et arabes, par exemple) sont, tout à la fois, profondément divisées et très versatiles. Il convient donc de rester prudent et ne pas tirer des plans sur la comète à partir de l'analyse de mouvements sociaux internationaux récents se développant dans un contexte mondial en évolution constante. Et ce, d'autant plus que l'histoire nous montre que l'influence des ONG sur la scène internationale n'est pas forcément plus grande aujourd'hui qu'au début du XXe siècle (Charnovitz, 2002). La forte visibilité médiatique des mouvements contestant la globalisation peut nous aveugler sur l'ampleur réelle de la mondialisation des actions protestataires. De plus, l'étude des organisations participant aux manifestations de Seattle, Porto Alegre ou Florence révèle que l'on assiste moins à une mondialisation de la société civile qu'à un rassemblement international hétéroclite d'organisations locales, 
nationales ou internationales. Il convient donc ne pas confondre l'objet de la mobilisation (la lutte contre la globalisation), le niveau territorial effectivement mobilisé (local, régional, national, continental, mondial) et le lieu de mise en visibilité de cette mobilisation (les contre-sommets du G8 ou de l'ONU). Pour le dire autrement, les organisations de la société civile réellement capables de mobiliser à l'échelon international sont, aujourd'hui, très peu nombreuses. De plus, ce type d'organisation internationale reste fortement marqué, dans sa structuration comme dans son mode d'action, par le cadre national qui leur a donné naissance (ollitrault, Baisnée, 1999). Par ailleurs, ces ONG soit disant mondiales, comme Greenpeace par exemple, ne sont présentes que dans une cinquantaine de pays qui, majoritairement, appartiennent à l'aire occidentale. De plus, ces grandes ONG occidentales ont généralement des modes d'organisation internes et des moyens d'expression externes qui les rapprochent plus des entreprises multinationales que des organisations démocratiques. Si l'on quitte le terrain empirique pour rejoindre celui de l'analyse théorique, l'hypothèse de l'émergence d'une société civile mondiale devient plus improbable encore. Ainsi, des auteurs comme M. Walzer (2000) et G. Eley (1992) soutiennent que la vision selon laquelle la société civile se développe contre les institutions politiques est par trop simpliste. Ils rappellent que dans les pays totalitaires le contrôle étatique empêche souvent le développement d'une société civile, l'histoire récente des pays d'Europe centrale illustre ce fait. De plus, en s'appuyant sur l'histoire de la démocratie française et américaine, ils montrent qu'il y a une interaction étroite entre le renforcement de la société civile et la démocratisation de l'État. C'est parce que l'État autorise un certain nombre de libertés publiques que peut se développer une société civile qui, en retour, pousse l'État à se démocratiser. Or, cette spirale vertueuse ne peut pas se développer à l'échelon mondial puisqu'il n'existe pas, à l'heure actuelle, un État mondial démocratique susceptible de favoriser l'émergence d'une société civile mondiale. Enfin, l'anthropologie politique (Abèles, 1988), nous montre que l'action politique, la participation à la vie publique, nécessite un cadre géographique dans lequel on peut mesurer et vérifier l'impact des décisions prises: il n'a pas de citoyenneté effective sans territoire clairement circonscrit. Pour toutes ces raisons, nous plaidons pour une analyse fine des phénomènes qui nous permettent de distinguer, d'un côté, l'émergence avérée de réseaux civiques internationaux comportant des organisations de tailles et de natures disparates qu'il convient d'analyser, de l'autre, un ensemble de discours politiques et idéologiques proclamant l'avènement d'une société civile internationale qu'il serait souhaitable de regarder avec circonspection. L'internationalisation de certains mouvements sociaux ne signifie pas obligatoirement naissance d'une société civile mondiale.

\section{BIBLIOGRAPHIE}

Abélès, M., (1988), « Anthropologie des espaces politiques français ", Revue française de science politique, vol. $38, \mathrm{n}^{\circ} 5$. 
Balme, R. et Chabanet, D., (2002), "Action collective et gouvernance de l'Union européenne », in : Balme R., Chabanet D. et Wright R., L'action collective en Europe, Paris, Presses de Sciences po.

Boual, J. C., (1999), Vers une société civile européenne, La Tour d'Aigues, Éditions de l'Aube.

Caillé, A., (2001), « La société civile mondiale qui vient », in : Laville J. L. et Caillé A. et al., Associations, démocratie et société civile, Paris, La découverte, 2001.

Calhoun, G., (1992), Habermas and the Public Sphere, Cambridge (Mass.), MIT Press.

CEDAG, (1998), Bilan annuel de la vie associative en Europe, Rapport dactyl., Paris, CEDAG.

Charnovitz, S., (2002), « Les ONG : deux siècles et demi de mobilisation », L'économie politique, n ${ }^{\circ} 13$.

Comité économique et social européen, (2000), La société civile organisée au niveau européen, Bruxelles, Comité économique et social européen.

Commission européenne, (2001), Gouvernance européenne, Bruxelles, Commission européenne.

Commission européenne, (1997), Communication de la Commission sur la promotion du rôle des associations et fondations en Europe, Bruxelles, Commission européenne.

Commission européenne, (1997b), Deuxième rapport sur la citoyenneté de l'Union, Bruxelles, Commission européenne.

Commission européenne, (1994), Croissance, compétitivité, emploi, Bruxelles, Commission européenne.

Doucin, M., (2001), Guide de la liberté associative dans le monde, Paris, La documentation française, 2001.

Eley, G., (1992), « Nations, Publics and Political Cultures », in : G. Calhoun, Habermas and the Public Sphere, Cambridge (Mass.), MIT Press.

Ferry, J. M., (1992), « Identité et citoyenneté européenne », in : J. Lenoble et N. Dewandre, L’Europe au soir du siècle, Paris, Éditions Esprit.

François, B. et Neveu, E., (1999), Espaces publics mosaïques, Rennes, PUR.

Fraser, N., (1992), «Rethinking the Public Sphere ", in : G. Calhoun, Habermas and the Public Sphere, Cambridge (Mass.), MIT Press.

Habermas, J., (1997), Droit et démocratie, Paris, Gallimard.

Habermas, J., (1987), Théorie de l'agir communicationnel, Paris, Fayard.

Habermas, J., (1978), L'espace public, Paris, Payot.

Laville, J. L., Caillé, A. et al., (2001), Associations, démocratie et société civile, Paris, La découverte.

Massiah, G., (2002), Le mouvement citoyen mondial, L'économie politique, $\mathrm{n}^{\circ} 13$.

Mouvement européen, (1997), Charte des citoyennes et citoyens européens, Bruxelles, Mouvement européen.

Mercier, A., (dir.), (2003), Vers un espace public européen ?, Paris, L'Harmattan.

Neveu, C., (1999), Espace public et engagement politique, Paris, L'Harmattan.

Ollitrault, S. et Baisnée, O., (1999), Comparaison franco-britanique du mouvement écologiste, Rapport de recherche programme « développement durable et systèmes écologiques ». 
Parlement européen, (1996), Résolution sur la participation des citoyens et des acteurs sociaux au système institutionnel de l'Union européenne, Strasbourg, Journal officiel des communautés européennes.

Peugeot, V., (1999), « Désir d’Europe », Transversales, n56.

Polanyi, K., (1983), La grande transformation, Paris, Gallimard.

Prometheus-Europe, (1999), Comment gérer l'Union européenne en 2020 ?, Paris, Centre Kléber.

Quéré, L., (1992), « L'espace public : de la théorie politique à la métathéorie sociologique », Quaderni, $n^{\circ} 18$.

Rangeon, F., (1986), « Société civile : histoire d'un mot » in : CURAPP, la Société civile, Paris, P.U.F.

Réau, E. du, (1995), L'idée d'Europe au XXe siècle, Bruxelles, Complexe.

Rebelle, B. et Swiatly, F., (1999), Libres associations, Paris, Desclée de Brouwer.

Ricœur, P., (1997), L'idéologie et l'Utopie, Paris, Seuil.

Rosanvallon, P., (1998), « Le nouveau travail de la représentation », Esprit, février.

Rousseau, D., (1999), « Pour une constitution européenne », Transversales, n56.

Salamon, L. M. et Anheier, H. K., (1994), The Emerging Sector, Baltimore, Johns Hopkins University.

Scholte, J. A., (2001), « Civil Society and Democracy in Global Governance », CSGR Working paper, $\mathrm{n}^{\circ} 65 / 01$.

Schudson, M., (1992), «Was There Ever a Public Sphere ? », in : G. Calhoun, Habermas and the Public Sphere, Cambridge (Mass.), MIT Press.

Smith, A., (1999), « L'espace public européen : une vue trop aérienne », Critique Internationale, n ${ }^{\circ} 2$.

Tassin, E., (1991), « Espace commun ou espace public ? », Hermès, nº10.

Walser, M., (2000), « Sauver la société civile », Mouvement, n8.

Weisbein, J., (2002), Construire la citoyenneté européenne, Thèse de science politique, Paris, IEP.

Weisbein, J., (2000), « La mobilisation autour de la citoyenneté de l’Union, l'exemple des associations européennes », Politique européenne, $n^{\circ} 2$.

Weisbein, J., (1998), « Construire la citoyenneté européenne ? Les contributions associatives à l'Europe politique », Communication au colloque « Action collective et intégration européenne », Oxford.

Wolton, D., (1993), La dernière utopie, Paris, Flammarion.

Worms, J. P., (1999), « Citoyenneté commune et différenciation culturelle », Hermès, n²3/24.

\section{NOTES}

1. www.vie-associative.gouv.fr.

2. La CPCA est un organe regroupant douze coordinations nationales couvrant les principaux domaines de la vie associative : le sport, l'éducation populaire, l'action sociale, etc.

3. L'Allemagne, l'Autriche, le Danemark, l'Italie, l'Irlande, la Grande-Bretagne et le Portugal. 
4. Ce nombre n'est pas spécifié dans des pays comme le Portugal ou la Suède ; ailleurs ce nombre varie entre deux (pour la France et les Pays-bas, par exemple) et 20 (Grèce).

5. Enquète qualitative menée, en 1997, sous l'égide du Centre européen des associations d'intérêt général (CEGAG, 1998).

6. Sport 127000 , Santé Loisir 82000 , Loisir Jeunnesse, 79000 : source Guide de la liberté associative dans le monde, p. 438.

7. C'est-à-dire des associations locales, nationales ou européennes militant pour le développement de la démocratie européenne.

8. Les auteurs ont compté 889 "eurogroupes" (associations spécifiquement regroupées au niveau européen) dont $3 \%$ (27) seraient des associations européennes centrées sur le thème de la citoyenneté (Balme, Chabanet, 2002, figure 5, p. 60). Ce chiffre n'est pas forcément contradictoire avec ceux du Forum permanent de la société civile, dans la mesure où, d'une part, ces 27 associations européennes peuvent être des réseaux regroupant de multiples associations locales ou nationales, et où, d'autre part, d'autres eurogroupes classés par les auteurs dans les rubriques «environnement ", "éducation culture ", " développement " pourraient être classés dans la catégories associations européennes civiques.

9. AGEE (Association des États généraux des étudiants d'Europe) pour les questions scolaires, COFACE (Confédération of Family Organizations of the European Community), pour les interrogations sur les droits familiaux, ECAS (Euro-Citizen Action-Service) dans le domaine de la citoyenneté et du droit des associations, EPHA (European Public Health Alliance) pour la santé, EWL (European Women's Lobby) dans le champs des droits des femmes, le Forum des migrants, pour les interrogations liées à l'immigration, IEIC (Inter Regional Institute for Consumer Protection) pour les questions touchant à la protection du consommateur.

10. Les CIC sont nées en 1996, elles s'articulent autour d'un «Info-centre " qui tient à jour un annuaire des partenaires des $\mathrm{CIC}$ et qui fait connaître à tous les membres du réseau les initiatives prises par l'un des leurs. Elles ont pour objectif principal de « Permettre que soit prise en compte la parole des citoyens au sein de la société civile dans la préparation et la révision des traités fondateurs de l'Union européenne" (extrait d'une plaquette de présentation). Les CIC sont membres du Forum permanent de la société civile.

11. Certains auteurs proposent, pour coller aux « réalités nouvelles », de donner une définition très large de la notion de "société civile » incluant les ONG, mais aussi les lobbies industriels et les organisations économiques du type chambre de commerce internationale (Scholte, 2001, par exemple). Ils s'appuient sur cette nouvelle définition pour conclure à l'émergence d'une société civile internationale qui viendrait combler le déficit démocratique engendré par la globalisation des marchés. Or, on le voit très bien en Europe, les lobbies économiques sont, de loin, les plus nombreux (Balme, Chabanet, 2002). Dès lors; s'appuyer sur le regroupement sous forme associative des acteurs économiques internationaux afin d'attester la naissance d'un contre pouvoir efficace à la globalisation des marchés mis en œuvre par ces mêmes acteurs économiques apparait, dans le meilleur des cas, comme un raisonnement purement tautologique.

\section{RÉSUMÉS}

Les faibles avancées institutionnelles de l'Union européenne ne doivent pas masquer la lente européanisation des associations. Or, les réseaux associatifs européens sont des acteurs politiques à part entière qui peuvent contribuer à l'émergence d'un espace public européen. Pour autant, 
doivent-ils être considérés comme les signes visibles de la construction d'une société civile internationale venant faire contrepoids à la globalisation financière ? Nous ne le croyons pas et plaidons, en conclusion, pour une analyse fine des phénomènes qui nous permettent de distinguer, d'un côté l'émergence effective de réseaux sociaux internationaux qu'il convient d'analyser, de l'autre un ensemble de discours politiques et idéologiques qu'il serait souhaitable de regarder avec circonspection.

The slow institutional progress of the European Union must not hide the creeping Europeanisation of associations. The network of European NGOs plays a full-time political role that can contribute to the birth of an European public sphere. But should they be considered as the visible signs of the construction of an international civil society that can act as a counterweight to financial globalisation? We do not think so. Therefore, in conclusion, we advocate a detailed analysis of certain features. Firstly, those that allow us to distinguish between the emergence of relevant international social networks and, secondly, the totality of political and ideological discourses which deserve to be looked upon with suspicion. Eric Dacheux : Associations et construction européenne : vers une société civile internationale?

\section{INDEX}

Mots-clés : Union Européenne, association, espace public, société civile, mobilisation

Keywords : European Union, association, public sphere, civil society, mobilization

\section{AUTEUR}

\section{ERIC DACHEUX}

CNRS (Laboratoire communication et politique) IUT de Roanne

Eric Dacheux est maître de conférences habilité à diriger les recherches en sciences de la communication à l'IUT de Roanne, et responsable de l'équipe Espace public européen du labo CNRS « Communication et Politique ». Ses derniers livres parus sont:

- L'Europe qui se construit : réflexions sur l'espace public européen, Presses universitaires de St Etienne, 2003.

- vaincre l'indifférence : le rôle des associations dans l'espace public européen, CNRS Éditions, 2000. 